\title{
Evolução das disparidades da extração vegetal e da silvicultura na Amazônia Legal: uma aplicação da cadeia de Markov
}

\author{
The development of differences in vegetal extraction and silviculture production in the legal amazon: \\ an application of Markov chains
}

\author{
Ricardo Bruno Nascimento dos Santos ${ }^{\mathrm{I}}$ Marcelo José Braga ${ }^{\mathrm{II}}$
}

\section{RESUMO}

\begin{abstract}
O artigo analisa a convergência municipal da produtividade vegetal (extração vegetal e silvicultura) na região da Amazônia Legal entre os anos de 1996 e 2006. Para analisar a convergência, optou-se pela metodologia da matriz de transição de Markov (Processo Estacionário de Primeira Ordem de Markov). Os resultados mostram a existência de 13 classes de convergência da produtividade vegetal. No longo prazo, a hipótese de convergência absoluta não se mantém, visto que 68,23\% dos municípios encontram-se numa classe inferior à média municipal, 33,54\% em uma classe intermediária acima da média e 13,41\% em uma classe superior acima da média.
\end{abstract}

Palavras-chave: modelo de Solow, matrizes de Markov, convergência.

\section{ABSTRACT}

This paper analyzes the convergence of the municipalities vegetable productivity in the Legal Amazon region between 1996 and 2006. The convergence analyses use the Markov transition matrix methodology (First Order Stationary Markov Process). The results show that existence of 13 classes of vegetable productivity convergence. In the long run, the hypothesis of absolute convergence is not maintained, whereas $68.23 \%$ of the municipalities are in a class lower than the municipal average, $33.54 \%$ in an intermediate class above average and $13.41 \%$ in a class higher than average.

Key words: Solow model, Markov matrices, convergence.

\section{INTRODUÇÃO}

Segundo o Instituto Brasileiro de Geografia e Estatística (IBGE), a produção da extração vegetal e da silvicultura somou, no ano de 2006, R \$ 10,9 bilhões (Informações extraídas da Pesquisa da Produção da Extração Vegetal e da Silvicultura). Desse total, 66\% são provenientes do segmento da silvicultura e $34 \%$ do extrativismo vegetal. Os Estados pertencentes à Amazônia Legal - AL (composta pelos Estados do Acre, Amapá, Amazonas, Maranhão, Mato Grosso, Pará, Rondônia, Roraima e Tocantins) - são responsáveis por 38,25\% da produção da Extração Vegetal e da Silvicultura (EV\&S) no Brasil. A região Nordeste (exclusive o Maranhão) é responsável por 22,64\%, seguida do Centro-Oeste (exclusive o Mato Grosso), com 14,29\%, e do Sul e Sudeste, com, respectivamente, $13,22 \%$ e $11,60 \%$.

A importância econômica de se estudar esse setor para a Amazônia Legal ocorre devido ao fato de ele estar ligado a uma importante cadeia produtiva na região, que é a do setor madeira e mobiliário, e também por se tratar de uma das principais fontes de matériaprima do setor no Brasil.

Segundo SANTANA (2002), um grande problema da região Norte é a prioridade que as pequenas e microempresas do setor madeira e mobiliário dão ao menor preço dos insumos. Considerando a qualidade como segunda opção, esse aspecto acaba influenciando numa menor valorização da produção vegetal (principalmente a madeira), que é abundante na região.

Uma forma de avaliar o crescimento, sobretudo econômico, de uma determinada atividade seria pelo uso do modelo de SOLOW (1956), que

'Programa de Pós-graduação em Economia, Faculdade de Economia, Universidade Federal do Pará (UFPA), 66075-110, Belém, PA, Brasil. E-mail: ricardobns@ufpa.br. Autor para correspondência.

IIDepartamento de Economia Rural, Universidade Federal de Viçosa (UFV), Viçosa, MG, Brasil. E-mail: mjbraga@ufv.br 
descreve o processo de crescimento equilibrado da economia, em que pressupõe uma função de produção, do tipo Cobb-Douglas, com rendimentos constantes à escala, em que o crescimento econômico é dado pela alocação dos fatores - capital (K) e trabalho (L), e por um fator de eficiência A, que representa a contribuição do trabalho na produção agregada, de forma que: $Y_{t}=F\left(K_{t}, A_{t} L_{t}\right)$. O conceito de convergência está baseado sobre a hipótese fundamentada nos modelos de crescimento neoclássico de Solow (1956), os quais mostram que as taxas de crescimento da renda ou do produto per capita de diferentes regiões geográficas tendem a se igualar ao equilíbrio de longo prazo. Segundo esses modelos, isso acontece porque há retornos decrescentes dos fatores produtivos e, por isso, uma região menos desenvolvida, que está utilizando menos intensamente esses fatores, tende a crescer mais rapidamente que uma região mais rica, alcançando o nível de renda ou de produção per capita desta no longo prazo. Tal hipótese equivale ao conceito de $\beta$-convergência absoluta, que ocorre quando independe das condições iniciais. A hipótese de convergência absoluta pode ser testada através de um modelo econométrico do tipo:

$$
\frac{1}{T} \ln \left(\frac{Y_{i, t+1}}{Y_{i, t}}\right)=\alpha+\beta \ln \left(Y_{i, t}\right)+\varepsilon_{i}
$$

em que $Y_{i, t}$ representa o PIB per capita da região $i$ no período inicial; $Y_{i, t+1}$ é o PIB per capita da região $i$ no período a frente; $T$ é o número de períodos analisados; $\alpha$ e $\beta$ são parâmetros estimados, $\varepsilon$ é o termo de erro, que deve ser aleatório. Diz-se que há $\beta$ convergência quando $\beta$ é negativo e estatisticamente significativo, indicando que a taxa média de crescimento da renda per capita entre os períodos $t$ e $t+1$ é negativamente correlacionada com o nível inicial de renda per capita.

Nesse contexto, este trabalho tem como objetivo analisar o processo de convergência da produtividade na produção vegetal na Amazônia Legal.

\section{MATERIAL E MÉTODOS}

O processo de convergência pode ser obtido da seguinte expressão:

$$
\mathrm{Y}_{\mathrm{t}+1}=\mathrm{MY}_{\mathrm{t}}
$$

em que $Y$ é um vetor linha de variáveis comparadas em dois períodos de tempo distintos (1996 e 2006); $M$ é a matriz de Probabilidades de Markov, cujos coeficientes, segundo SIMON \& BLUME (2004, p.617), representam a probabilidade de passagem de um estado para outro. Para que o sistema tenha soluções, é necessário estudar as propriedades dessa matriz.
A matriz de probabilidades de transição de níveis de produtividade vegetal, entre os municípios da Amazônia Legal, é construída pelo uso da razão dessa variável em relação à média dos municípios, a qual será considerada igual à unidade.

No entanto, um importante aspecto é a definição do intervalo de classe que será utilizado para a construção da matriz de Markov, além de verificar se os dados de produtividade vegetal (1996-2006) possuem o comportamento de uma distribuição normal para os municípios da AL. Os testes de normalidade são necessários, uma vez que a construção das classes de níveis de produtividade vegetal requer a hipótese de normalidade da distribuição dos dados populacionais de onde foi extraída a amostra necessária para estabelecer a amplitude da classe de nível de valor da produção, a qual será denominada $h$.

O valor de $h$, ou seja, a amplitude de classe é importante para a estimativa da função densidade de probabilidade. Existe um trade-off para a escolha de $h$. Segundo PAGAN \& ULLAH (1999), uma amplitude de classe muito grande faz com que haja grande número de pontos em cada intervalo, diminuindo a variância da estimativa, que produz um viés da estimativa do histograma de frequência. Com uma amplitude de classe pequena, ocorre o contrário: reduz-se o viés, mas a variância aumenta. Dessa forma, o valor de $h$ deve ser escolhido para se fazer uma escolha ótima para o tradeoff entre viés e variância da estimativa.

Conforme MAGRINI (1999), há três critérios para a definição de $h$. Esses critérios, que podem ser vistos com mais detalhes em DEVROYE \& GYORF (1985), FREEDMAN \& DIACONIS (1981) e SCOTT (1979), também podem ser utilizados em distribuições não normais; assim, o valor ótimo do intervalo de classe é dado por $h=2,72 \mathrm{sn}^{-1 / 3}$, em que $s$ é o desvio-padrão da distribuição e $n$, o número de observações.

Encontradas as classes do valor da produtividade vegetal, pode-se estimar a matriz de transição (de Markov), por meio de um estimador de máxima verossimilhança da probabilidade de transição, comparando o número de municípios que pertencem a certa classe do período de 1996 e migram para outras classes, ou permanecem nela, em 2006. As probabilidades de transição podem ser estimadas por:

$$
\hat{P_{i j}}=\frac{\sum_{k=1}^{n} I_{A j}\left(X_{t+1, k}\right) I_{A k}\left(X_{t, k}\right)}{\sum_{k=1}^{n} I_{A i}\left(X_{t, k}\right)}
$$

em que $\hat{P}_{i j}$ é a probabilidade de transição da classe $i$, no período $t$ (1996), para a classe $j$, no período $t+1$ (2006); $A i$ corresponde à classe da produtividade vegetal em 1996; $A j$ é a classe da produtividade para o ano de 
2006; X são os elementos da distribuição; e I(.) é a função de contagem.

A matriz de Markov relaciona o vetor de distribuição de probabilidade da variável produtividade vegetal nas $h$ classes $A i$, no período $t$, com aquela verificada no período $t+1$. A diagonal principal representa uma probabilidade de o município permanecer na mesma classe, e os elementos fora da diagonal principal representam a probabilidade de transição entre as classes.

Segundo BICKENBACH \& BODE (2002), pode-se afirmar que haverá convergência quando a norma dos autovalores reais ou complexos de $M$ for menor que a unidade. Como a soma das linhas da matriz de Markov $M$ tem que ser 1, pois se trata de uma matriz de probabilidade, obtém-se sempre um autovalor 1. Portanto, sempre haverá convergência para um ou mais vetores de distribuição da produtividade vegetal. A presença de um único autovalor unitário e dos demais com norma menor que 1 indica que há um processo de convergência para uma única distribuição de probabilidade, que será linear no autovetor correspondente ao autovalor unitário. Com esse vetor de convergência, pode-se descrever a estrutura da distribuição da produtividade vegetal.

Assim como as demais metodologias para teste de convergência, essa também apresenta deficiências, cabendo destacar que o processo estacionário de primeira ordem, de Markov, assenta-se na hipótese teórica de rendimentos marginais decrescentes. Além disso, não reconhece a possibilidade de os choques exógenos afetarem o curso da produtividade vegetal até o estado estacionário, visto que considera a probabilidade de transição constante ao longo do tempo. Para um sistema de equações em diferenças de primeira ordem, a distribuição de probabilidade para k períodos à frente depende unicamente de uma potência da matriz de probabilidades de transição, $M: Y_{t+k}=M^{k} Y^{t}$. A distribuição de probabilidade ergótica, definida no estado estacionário, ocorre quando $k \rightarrow \propto$.

Ainda nessa perspectiva, segundo STÜLP \& FOCHEZATTO (2004), pode-se estimar a velocidade com que o equilíbrio de longo prazo é alcançado por meio da segunda raiz característica. Essa velocidade representa o tempo necessário para percorrer a metade da distância entre a posição inicial e a de equilíbrio de longo prazo $(\mathrm{dm})$, denominado na literatura de meiavida. Algebricamente, tem-se $d m=-\log 2 / \log \left|\lambda_{2}\right|$, em que 12 é o segundo maior autovalor.

Os dados utilizados para compor a produtividade vegetal foram o valor da produção extrativa vegetal mais o valor da produção da silvicultura, coletados no banco de dados agregados do Sistema IBGE de Recuperação Automática (Sidra) para o ano de 1996 e 2006. Para compor a variável per capita, o valor da produção foi dividido pelo número do pessoal ocupado nos dois setores.

As informações geradas não passaram pelo teste de normalidade, no entanto, seguindo CHESHIRE \& MAGRINI (2000), foi utilizado o procedimento de DEVROYE \& GYORF (1985) para o cálculo do valor de $h$, sendo passível de uso a dados não normais.

Dos 763 municípios da Amazônia Legal, foram utilizados neste trabalho 424 municípios da Amazônia Legal, de acordo com a definição do Instituto de Pesquisa Econômica Aplicada. Muitos municípios ficaram de fora pela falta de informações para o ano de 1996.

\section{RESULTADOS E DISCUSSÃO}

Os resultados para os 424 municípios da Amazônia Legal indicaram um valor de $h$ de 0,44 , o qual não se mostrou adequado, pois não contemplava todas as observações da distribuição. Optou-se, assim, por um valor arbitrário de 0,50 , que melhor se ajustava aos dados, definindo as 13 classes. As distribuições do valor da produtividade da produção vegetal foram normalizadas pelo desvio em relação à média dos municípios da Amazônia Legal. A ideia desse procedimento é possibilitar a classificação das duas distribuições (1996 e 2006) em um mesmo intervalo de classe, conforme apresentado na tabela 1.

O valor médio da produtividade vegetal dos municípios pertencentes a Amazônia Legal foi de aproximadamente R\$3.231 em 1996 e R\$ 1.559 em 2006. De acordo com os resultados, é possível observar que existem 13 classes do valor da produção agropecuária. A classe mais pobre é a 1 , que vai de $0,9 \%$ da média até $50 \%$ da média. A classe 2 vai de $50 \%$ da média até a média. A classe 13 é a mais rica, indo de $600 \%$ da média até $652 \%$ desta.

Na tabela 2, encontra-se o percentual de municípios que melhoraram e pioraram de classe entre os anos de 1996 e 2006. Pelos resultados, fica evidente que o número de municípios que melhoraram de posição é um pouco maior que o daqueles que pioraram. Observa-se que 144 municípios pioraram de posição, 149 melhoraram e 131 mantiveram sua posição na classe entre os anos de 1996 e 2006. Uma tendência estadual de melhora ou piora pode ser observada na subtabela da figura 1.

Pela estrutura da matriz de probabilidade de transição (Markov), observa-se uma tendência de convergência para classes de menor renda. Calculouse a distribuição de probabilidades para o estado 
Santos \& Braga.

Tabela 1 - Classes do valor da produtividade vegetal, distribuição das classes e o estado estacionário para os municípios da Amazônia Legal (1996/2006).

\begin{tabular}{lcccc}
\hline Classes do valor da produção & Limite de Classe & Distribuição em 1996 & Distribuição em 2006 & Estado Estacionário \\
\hline Classe 1 & 0,009 a 0,5 & 45,99 & 45,99 & 45,93 \\
Classe 2 & 0,5 a 1,0 & 22,64 & 21,23 & 22,30 \\
Classe 3 & 1,0 a 1,5 & 10,61 & 13,21 & 10,95 \\
Classe 4 & 1,5 a 2,0 & 7,31 & 5,90 & 7,18 \\
Classe 5 & 2,0 a 2,5 & 3,77 & 3,07 & 3,73 \\
Classe 6 & 2,5 a 3 & 3,07 & 3,30 & 3,17 \\
Classe 7 & 3,0 a 3,5 & 1,89 & 1,65 & 1,98 \\
Classe 8 & 3,5 a 4,0 & 0,94 & 1,18 & 0,91 \\
Classe 9 & 4,0 a 4,5 & 0,94 & 0,94 & 0,89 \\
Classe 10 & 4,5 a 5,0 & 0,47 & 0,71 & 0,50 \\
Classe 11 & 5,0 a 5,5 & 0,94 & 1,18 & 0,77 \\
Classe 12 & 5,5 a 6,0 & 0,71 & 1,18 & 0,71 \\
Classe13 & 6,0 a 6,52 & 0,71 & 0,47 & 0,76 \\
\hline
\end{tabular}

Fonte: Elaboração dos autores.

estacionário, que corresponde à solução do sistema de equações em diferenças.

A partir dos resultados da tabela 2, determinou-se a evolução temporal da distribuição da produtividade vegetal, que pode ser vista na figura 1 . Entre 1996 e 2006, observa-se que os municípios estavam, em sua maior parte, concentrados nas classes 1 e 2, com, respectivamente, 45,99\% e 21,93\% da distribuição. A classe que mais cresceu foi a 3, passando de $10,61 \%$ para $13,21 \%$. As classes com a maior queda foram a 2 e a 4, ambas caindo 1,42 ponto percentual de 1996 a 2006. No estado estacionário, o qual consiste na solução do sistema de equações em diferença, observase que, no longo prazo, os municípios estariam migrando para as classes mais intermediárias, principalmente para as classes 4 e 7 .

Além disso, observa-se, pela distribuição da produtividade vegetal, que a hipótese de convergência absoluta não se mantém, o que era de se esperar, dada a heterogeneidade dos estados estacionários dos itens da amostra. O cálculo da velocidade de convergência foi de 0,274 no período, o que representa, nos termos do corte temporal em que foi feita a distribuição do valor da produtividade vegetal, aproximadamente 5,35 anos para os setores de extração vegetal e silvicultura percorrer a metade da distância entre a posição inicial e a de equilíbrio de longo prazo.

Na tabela 1, percebe-se que as mudanças de classes entre os municípios da Amazônia Legal são equilibradas, porém elas podem estar ocorrendo na melhora de alguns municípios e piora de outros. Na figura 1, podem-se identificar, espacialmente, quais Estados tornaram-se mais pobres no setor EV\&S e quais evoluíram entre os anos de 1996 e 2006.

O Estado que mais evoluiu no setor EV\&S foi o Tocantins (TO), que apresentou a segunda maior melhora absoluta. Junto com o Pará, ele teve o maior número de municípios que melhoraram (34), o que corresponde a 55,74\% dos 61 municípios que fizeram parte da amostra. Também foi um dos Estados que apresentaram um dos menores valores absolutos de

Tabela 2 - Aumento (diminuição) relativo(a) dos municípios da Amazônia Legal entre os anos de 1996 e 2006.

\begin{tabular}{lcccc}
\hline Número de posições & Melhoraram & \% da Amostra & Pioraram & \% da amostra \\
\hline Entre 11 e 6 & 21 & 3,7 & 16 & 2,8 \\
Entre 5 e 2 & 67 & 11,9 & 70 & 12,5 \\
1 & 61 & 10,8 & 58 & 10,3 \\
0 & 131 & 23,3 & - & - \\
Total & 280 & 49,8 & 144 & 25,6 \\
\hline
\end{tabular}

Fonte: Elaboração dos autores. 


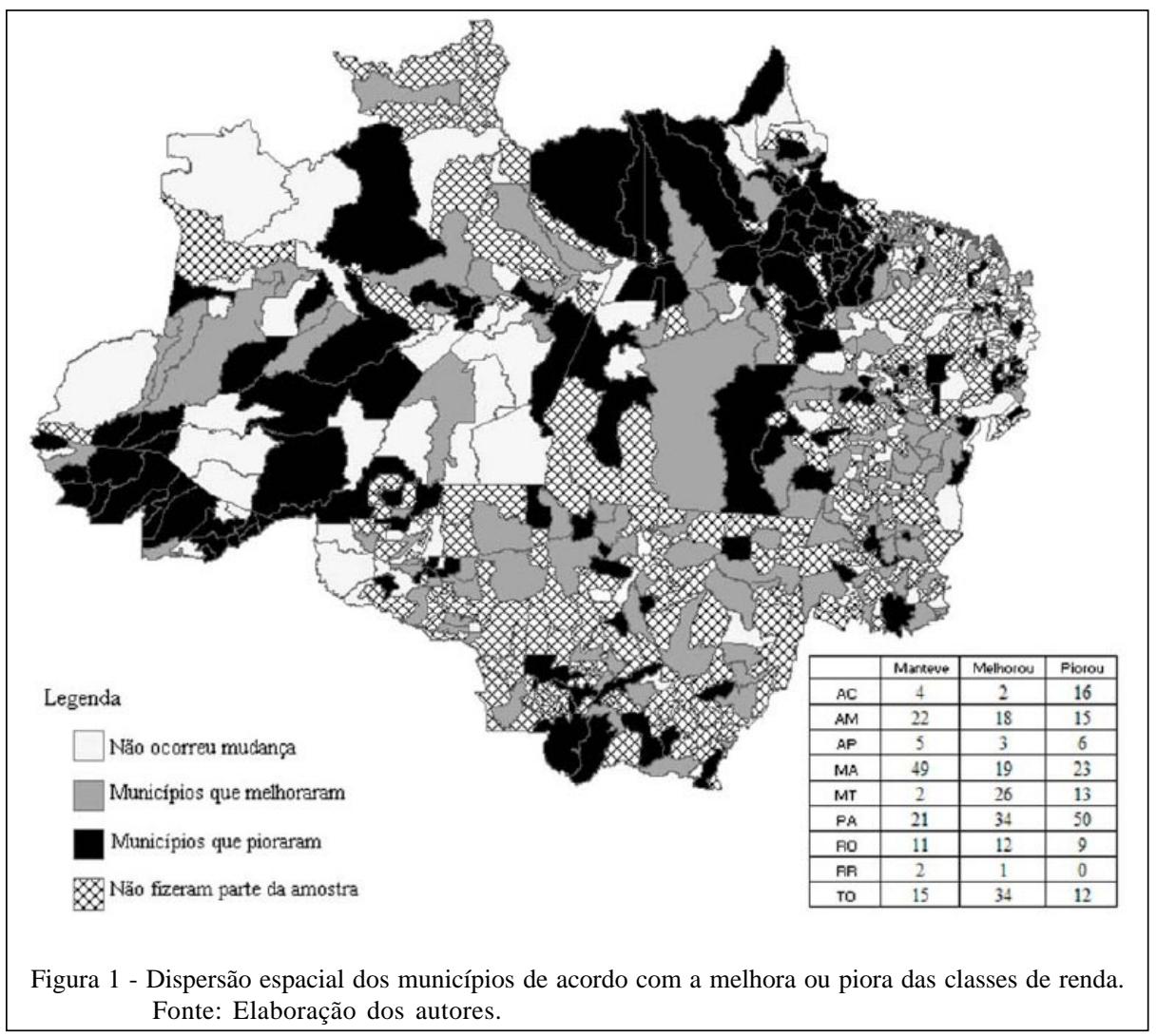

piora, com 12 municípios, que representam $19,67 \%$ dos municípios do estado de TO. Dessa forma, dentro do processo de convergência de longo prazo, será o município mais beneficiado no setor EV\&S. O Mato Grosso (MT) teve o segundo melhor desempenho: dos seus 41 municípios que fizeram parte da amostra, 2 $(4,88 \%)$ mantiveram-se na mesma classe de produtividade vegetal, enquanto 26 (63,41\%) melhoraram de classe e 13 (31,71\%) pioraram. Um dos elementos que explica a melhora nesses estados é o intenso desenvolvimento que neles ocorre, muito por conta de serem unidades federativas que passaram por processo de separação. O governo do estado de Tocantins tem feito políticas de reflorestamento e de produção de energia a partir da silvicultura e de outras formas de energias renováveis.

Em se tratando dos municípios que tiveram pior desempenho, destacam-se o Acre, onde 16(72,73\%) dos municípios da sua amostra pioraram, e o Pará, que, em termos absolutos, teve o maior número de municípios que pioraram (50), com 47,62\% de sua amostra. O terceiro pior desempenho foi o do Amapá, onde 15 (42,86\%) dos municípios pioraram de classe entre 1996 e 2006. No Pará, o principal fator para o pior desempenho entre os estados é a burocracia. A demora nos licenciamentos ambientais e dos planos de manejo e reflorestamento, que são fontes seguras de matéria prima madeireira. Tais barreiras impedem que empresas ampliem seus investimentos no setor.

\section{CONCLUSÃO}

A teoria econômica e as evidências empíricas têm mostrado a possibilidade de convergência entre regiões, porém as recentes evidências contestam a possibilidade de convergência absoluta. Os resultados obtidos neste trabalho com a variável produtividade vegetal, no decênio 1996-2006, também invalidam a hipótese de convergência absoluta dos municípios da Amazônia Legal.

A metodologia empregada permitiu identificar a dinâmica distributiva do setor extração vegetal e silvicultura no longo prazo. No estado estacionário, a maior parte dos municípios da $\mathrm{AL}$ permanece no mesmo nível de classe, estando a maioria deles concentrada na parte inferior da distribuição (classes 1, 2 e 3). Isso mostra que o crescimento econômico, por si só, não está conseguindo amenizar as disparidades existentes entre os municípios da AL, no que tange ao valor da produção vegetal. 
Conclui-se que as estimativas apontam para um padrão de convergência ruim, no sentido de que os municípios, em sua maioria, não estão conseguindo, em média, criar trajetórias de crescimento sustentado que os levem para classes superiores de valor da produção. Em outras palavras, no período analisado, o hiato de crescimento da produtividade vegetal permaneceu estável, sem significativas melhoras.

\section{REFERÊNCIAS}

BICKENBACH, F.; BODE, E. Markov or not Markov - This should be a question. In: CONGRESS OF THE EUROPEAN REGIONAL SCIENCE ASSOCIATION, 2002. Dortmund. Proceedings... Dortmund: ERSA, 2002. p.27-31. Disponível em: <http://www-sre.wu-wien.ac.at/ersa/ersaconfs/ersa02/cdrom/papers/024.pdf>. Acesso em: 03 jan. 2010.

CHESHIRE, P.; MAGRINI, S. Endogenous processes in European Regional growth: convergence and policy. Growth and Change, v.31, p.455-479, 2000. Disponível em : <http:/ /www.blackwell-synergy.com/doi/abs/10.1111/00174815.00140>. Acesso em: $26 \mathrm{dez}$. 2009. doi: 10.1111/00174815.00140 .

DEVROYE, L.; GYORF, L. Nonparametric density estimation: the L1 view. New York: John Wiley, 1985. 386p.

FREEDMAN, D.; DIACONIS, P. On the histogram as a density estimator: L2 theory. Zeitschrift fur Wahrscheinlichkeitstheorie und verwandte Gebeite, v.57, p.453-476, 1981. Disponível em: $<$ http://www.mendeley.com/research/on-the-histogram-as-a-densityestimatorl2-theory/\#>. Acesso em: 14 dez. 2009. doi: 10.1007/ BF01025868.
MAGRINI, S. The evolution of income disparities among the regions of the European Union. Regional Science and Urban Economics, v.29, n.2, p.257, 281, 1999. Disponível em: <http: //www.sciencedirect.com/science/article/B6V89-3VJ2WMT-7/2/ 31147ddf8293a95118365cb543c928c1>. Acesso em: 03 jan. 2010. doi: 10.1016/S0166-0462(98)00039-8.

PAGAN, A.; ULlah, A. Nonparametric econometrics. Cambridge: Cambridge University, 1999. 444p.

SANTANA, A.C.D. A competitividade sistêmica das empresas de madeira da região Norte. Belém: M\&S, 2002. 304p.

SCOTT, D.W. On optimal and data-based histograms. Biometrika, v.66, p.605-610, 1979. Disponível em: <http:/ /biomet.oxfordjournals.org/content/66/3/605.full.pdf+html>. Acesso em: 20 jan. 2010. doi: 10.1093/biomet/66.3.605.

SIMON, C.P.; BLUME, L. Matemática para economistas. Porto Alegre: Bookman, 2004. 920p.

SOLOW, R.M. A contribution to the theory of economic growth. Quarterly Journal of Economics, v.70, n.1, p.65-94, 1956. Disponível em: <http://qje.oxfordjournals.org/content/70/1/ 65.full.pdf+html>. Acesso em: 12 mar. 2010. doi:10.2307/ 1884513

STÜLP, V.J.; FOCHEZATTO, A. A evolução das disparidades regionais no Rio Grande do Sul: uma aplicação de matrizes de Markov. Nova Economia, v.14, n.1, p.39-66, 2004. Disponível em: <http://www.face.ufmg.br/novaeconomia/sumarios/v14n1/ Stulp.pdf $>$. Acesso em: 03 mar. 2010. 